\title{
Statyba
}

\section{DISTORSION IN STRUCTURAL MECHANICS}

\section{J. Atkočiūnas}

To cite this article: J. Atkočiūnas (1995) DISTORSION IN STRUCTURAL MECHANICS, Statyba, 1:1, 3-24, DOI: $10.1080 / 13921525.1995 .10531499$

To link to this article: https://doi.org/10.1080/13921525.1995.10531499

Published online: 26 Jul 2012.

Submit your article to this journal

Џ Article views: 102 


\section{ДИСТОРСИЯ В СТРОИТЕЛЬНОЙ МЕХАНИКЕ}

\section{Ю.Ю. Аткочюнас}

\section{1. Введение}

Методические положения о расчете усилий, деформаций и перемещений упругих конструкций при заданных нагрузках $\mathbf{F}$ и разных внешних воздействиях (задача анализа) в строительной механике хорошо известны [1]. Дисторсия q - это деформация, возбуждаемая факторами, не связанными с регулярным геометрически совместным деформированием конструкции. Она может быть обусловлена разными причинами: изменениями температуры $t$, осадкой опор $u_{c}$, предварительным напряжением, дефектами изготовления (пластические деформации $\Theta_{p}-$ своего рода пример дисторсии) [2-4]. Понятие дисторсии может выступить связуюшим звеном как в пределах упругого расчета системы при разных видах воздействий, так и для упругой и упругопластической стадий работы самой конструкции. Появляется возможность построения единой (полной) матричной системы уравнений решения задачи анализа упругих и упругопластических строительных конструкций при заданных внешних нагрузках $\mathbf{F}$ и воздействиях q ( деформациях $\Theta_{p}$ ). В настоящей работе единая система уравнений получена и на основе экстремальных энергетических принципов [4-7] . Приводятся не связанные с конкретным методом дискретизации математические модели экстремальных задач анализа, в том числе и приспособляющихся конструкций при наличии дисторсии ( для коэффищиента запаса по приспособляемости близкого $\mathrm{k}$ 1). При алгоритмизации решения упомянутой единой системы уравнений могут использоваться разные методы: метод конечных разностей, метод равновесных конечных элементов (МКЭ; основные неизвестные - усилия; удовлетворяются уравнения равновесия) [8-13]. Здесь возможно использование хорошо разработанных процедур формирования матриц жесткости и податливости равновесных элементов. Тогда появляется - и это важное качество дисторсии - возможность использования полученных “упругих" элементных матриц для анализа конструкции и в ее пластической стадии работы. 
Приведем несколько общих замечаний о материале настоящей статьи. В нашей работе [5], имеющей прямое отношение только $\mathrm{k}$ теории расчета идеально упругопластических систем при повторных нагружениях, попугно высказана и общая идея о возможности применения дисторсии в строительной механике (без аллюзии на детальные алгоритмы конкретных методов дискретизации). В настоящей работе на основе экстремальных энергетических принципов и делается определенная попытка дальнейшего развития работ [14-16]. При этом важным обстоятельством является то, что в качестве основных неизвестных при наличии дисторсии привлекаются, как правило, не суммарные усилия, деформации и перемещения, а самоуравновешенные (в том числе и остаточные) составляющие напряженно-деформированного состояния (НДС) конструкции. Только в этом случае непосредственно можно учесть существующие в конструкции пластические деформации как единственный фактор внешних воздействий. Это становится очевидным при анализе минимального принципа Койтера для суммарньх напряжений [6]. При подстановке заданных пластических деформаций в отвечающие этому принципу математические выражения получается минимальный принцип Койтера для остаточных напряжений, не требующий указания конкретных причин возникновения пластических деформаций [6]. Построение задач расчета НДС в терминах остаточных его составляюших значительно облегчает разработку шаговых методов анализа упругопластических конструкций [17].

\section{2. Единая система матричных уравнений}

НДС упругой конструкции анализируется решением известной системы уравнений (традиционно в строительной механике она приводится для дискретной модели в целом):

$$
\begin{aligned}
& {[A] \mathbf{S}_{e f}=\mathbf{F},} \\
& {[A]^{T} \mathbf{u}_{e f}=\Theta_{e f},} \\
& \Theta_{e f}=[D] \mathbf{S}_{e f} .
\end{aligned}
$$

Искомыми здесь выступают $n$-мерные векторы усилий $\mathbf{S}_{e f}$, деформаций $\Theta_{e f}$ и $m$ - мерный вектор перемещений $\mathbf{u}_{e f}$ упругого расчета (степень статической неопределимости $k_{0}=n-m$; индекс $e$ относится $\mathrm{x}$ абсолютно упругой системе, а $f$ указывает на то, что составляющие НДС рассчитаны от внешней нагрузки F). Здесь $[A]-m \times n-$ мерная матрица коэффициентов уравнений равновесия, $[D] \quad-$ квазидиагональная матрица податливости. Решение системы (1)-(3) представляется выражениями: 


$$
\begin{aligned}
& \mathbf{u}_{e f}=\left(|A|[I]^{-1}[A]^{T}\right)^{-1} \mathbf{F}:=[(I]] \mathbf{F} \\
& \left.\mathbf{S}_{e f}=[D]^{-1}[A]^{I}(\mid A][D]^{-1}[A]^{I}\right)^{-1} \mathbf{F}=[\iota] \mathbf{F}
\end{aligned}
$$

Зљесь $|\beta|^{-1}=\left[A \mid[D]^{-1}[A]^{T}-\right.$ матриґа жесткости конструкции (в метоце конечнғх

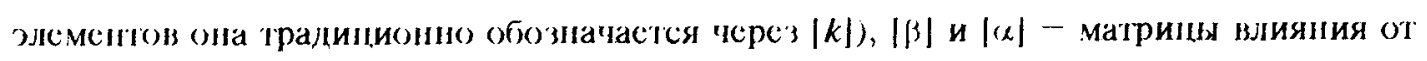
li. И'з уравнсний равновесия

$$
\left[A_{c}\right] \mathbf{S}_{e f}=\mathbf{R}_{e f}
$$

онредсляктся олорныс рсакции $\mathbf{R}_{\text {ef }}$

$$
\mathbf{R}_{e f}=\left[A_{C}\right][(\alpha] \mathbf{F}
$$

Уравнсния совместности $[B] \Theta_{e f}=\mathbf{0}$ лия деформаций $\Theta_{e f}$ получаются из геомстрических уравнений (2) исключением из них перемешений $\mathbf{u}_{e f}$. Здесь матрица $[B]=\left[\left[A^{\prime \prime}\right]^{T}\left(\left[A^{\prime}\right]^{T}\right)^{-1},-[1]\right]$ зависит только о'т геометрии конструкции и действительна для упругой и упругопластической стадий ее работы (в рамках принимаемых для идеальной пластичности геометрических допущений). При этом считается, что для подматрицы $\left[A^{\prime}\right]$ суцествует $\left[A^{\prime}\right]^{-1}$. Деформации элементов конструкции, являющиеся составляюшцми вектора $\mathbf{q}$, лля которого $[B] \mathbf{q} \neq \mathbf{0}$, в настоящей работе и относятся $\mathrm{k}$ понятию дисторсии. Понятие дисторсии не является строго регламентированным (под дисторсией можно понимать не только деформащии как таковые, но, и например, заданные перемещения или осадки опор; возможен и традиционный - смешанный подход). В настоящей работе с целью построения единой матричной системы уравнений решения задачи анализа упругих и упругопластических конструкций под дисторсией понимаются только деформации.

Дисторсия $q$ может быть интерпретирована и как сумма деформаций $\mathbf{q}=\Theta_{q t}+\Theta_{q e}+\Theta_{q c}$. Объясним подробнее. Для закрепленного элемента дискретной модели конструкции подбирается основная система. В расчетных сечениях основной системы по известным формулам, таблицам или с использованием данных соответствуюних конечных элементов - это зависит от сложности исследуемой конструкции - определяются деформации от температурных воздействий и предварительного напряжения. Так определяются векторы $\Theta_{q t}$, $\Theta_{q e}$ для дискретной модели конструкции в целом. Здесь $\Theta_{q t}$ - вектор деформаций элементов дискретной 
модели конструкции от температурных изменений $t \cdot \Theta_{q e}-$ деформации от предварительного напряжения. $\Theta_{q c}$ - деформации от заданного смещения опор $\mathbf{u}_{c}$ :

$$
\Theta_{q c}=\left[A_{c}\right]^{T} \mathbf{u}_{c} .
$$

В конструкции дисторсия q (или пластические деформации) обуславливает возникновение самоуравновешенных усилий $\mathbf{S}_{e q}$ и перемещений $\mathbf{u}_{e q}$ :

$$
[A] \mathbf{s}_{e q}=\mathbf{0},[A]^{T} \mathbf{u}_{e q}=\Theta_{e q}, \Theta_{e q}=[D] \mathbf{s}_{\epsilon, q}+\mathbf{q}
$$

где деформации $\Theta_{e q}=[D] \mathbf{S}_{e q}+\mathbf{q} \quad$ совместны, т.е. $[B] \Theta_{e q}=[B]\left([D] \mathbf{S}_{e q}+\mathbf{q}\right)=\mathbf{0}$. Решением системы уравнений (8) относительно $\mathbf{u}_{e q}, \mathrm{~S}_{e q}$ (без рассмотрения конкретных причин возникновения дисторсий q) получается система матричных уравнений [14-16]:

$$
\begin{aligned}
\mathbf{u}_{e q} & =\left([A][D]^{-1}[A]^{T}\right)^{-1}[A][D]^{-1} \mathbf{q}=[\alpha]^{T} \mathbf{q}=[\bar{H}] \mathbf{q}, \\
\mathbf{S}_{e q} & =\left\{[D]^{-1}[A]^{T}\left([A][D]^{-1}[A]^{T}\right)^{-1}[A][D]^{-1}-[D]^{-1}\right\} \mathbf{q}= \\
& =\left\{[\alpha][A][D]^{-1}-[D]^{-1}\right\} \mathbf{q}=[\bar{G}] \mathbf{q} .
\end{aligned}
$$

Физический смысл произведения $[A][D]^{-1} \mathbf{q}=\mathbf{F}_{q}-$ нагрузка, равнодействующая дисторсным воздействиям. Например, для температурного изменения узловые усилия элементов $\mathbf{s}_{q t}$ могут быть определены по зависимости $\mathbf{s}_{q t}=[D]^{-1} \Theta_{q t}$ и в конечном счете $[A] \mathbf{S}_{q t}=\mathbf{F}_{q t}$.

Объединением зависимостей (4), (5) и (9), (10) получается единая система расчета усилий $\mathrm{S}_{e}$, перемещений $\mathbf{u}_{e}$ и деформаций $\Theta_{e}$ от заданных нагрузки $\mathrm{F}$ и дисторсий q:

$$
\begin{aligned}
& \mathbf{u}_{e}=\mathbf{u}_{e f}+\mathbf{u}_{e q}=[\beta] \mathbf{F}+[\bar{H}] \mathbf{q}, \\
& \mathbf{S}_{e}=\mathbf{S}_{e f}+\mathbf{S}_{e q}=[\alpha] \mathbf{F}+[\bar{G}] \mathbf{q} .
\end{aligned}
$$

Опорные реакции $\mathbf{R}_{e}$, используя зависимости (6), (7) и (11), рассчитываются по формуле:

$$
\mathbf{R}_{e}=\left[A_{c}\right]([\alpha] \mathbf{F}+[\bar{G}] \mathbf{q}) .
$$

Формулы (11) значительно упрощаются, если вектор $\mathbf{F}=\mathbf{0}$. Перемещения и усилия в этом случае обуславливаются только дисторсией q. В этом случае получаются уравнения равновесия для самоуравновешенной системы: 


$$
[A] \mathbf{S}_{e}=\mathbf{0} .
$$

Это нетрудно показать, анализируя $\mathbf{S}_{e}$ согласно формуле (11), например, для случая предварительного деформирования:

$$
\mathbf{S}_{e}=[\bar{G}] \mathbf{q}=\left\{[\alpha][A][D]^{-1}-[D]^{-1}\right\}[D] \mathbf{S}_{q e}=[\alpha][A] \mathbf{S}_{q e^{-}}-[\mathrm{I}] \mathbf{S}_{q e}
$$

и, применяя оператор [A], получаем

$$
[A] \mathbf{S}_{e}=[A]\left([\alpha][A] \mathbf{S}_{q e}-\mathbf{S}_{q e}\right)=[A] \mathbf{S}_{q e}-[A] \mathbf{S}_{q e}=\mathbf{0} .
$$

При наличии пластических деформаций в формулах (11) $\mathbf{S}_{e}$ и $\mathbf{u}_{e}$ не являются усилиями и перемещениями упругого расчета и поэтому часто называются суммарными усилиями $\mathbf{S}$ и перемещениями и. Усилия, обусловленные пластическими деформациями $\Theta_{p}$ и в прямом смысле зтого слова являющиеся остаточными, т.е. существуюшими и удовлетворяющими уравнениям равновесия при $\mathbf{F}=\mathbf{0}$, принято обозначать через $\mathbf{S}_{r}$, а остаточные перемещения - через $\mathbf{u}_{r}$ (эти обозначения будут использоваться и для задач математического программирования, построенных на основе принципов минимума дополнительной и полной потенциальной энергий). На формулы (9), (10) индекс $r$ не распространяется, так как при удалении внешпнего воздействия $\mathbf{q}$ самоуравновешенные усилия $\mathbf{S}_{e q}$, перемещения $\mathbf{u}_{e q}$, деформации $\Theta_{e q}$ исчезают. Однако матрицы $[\bar{H}]$ и $[\bar{G}]$, входящие в формулы расчета конечных значений составляющих НДС конструкции (9),(10) ках известно, формально одинаковы для упругой и упругопластической стадий работы.

Поэтому заданные пластические деформации $\Theta_{p}^{*}$ в дальнейшем отождествляются с дисторсией $\mathbf{q}=\Theta_{p}^{*}$, а $\mathbf{S}_{e q}=\mathbf{S}_{r}^{*}, \mathbf{u}_{e q}=\mathbf{u}_{r}^{*}$.

Матрица $[\bar{G}]$ является вырожденной, т. е. не существует ее обратной матрицы. Неизвестные $\mathrm{S}_{e q}$ и $\mathbf{u}_{e q}$ являются однородными функциями дисторсий (в том числе от заданных $\Theta_{p}^{*}$ ). Поэтому для $\mathbf{S}_{e q}$ и $\mathbf{u}_{e q}$ действителен принцип суперпозиции и, например, для состояния приспособляемости упругопластической конструкции возможно применение принципа Бэтти $[2,3]$.

Определение пластических деформаций $\Theta_{p}$ от внешних нагрузок и разных воздействий в свою очередь является отдельной проблемой механики диссипативных систем. В контексте понятия дисторсии этот вспрос более подробно будет рассматриваться в заключительных разделах работы. Здесь же для анализа 
НДС упругих и упругопластических систем по формулам (9), (10) принимается, что $\Theta_{p}^{*}$-известный вектор.

\section{3. Дисторсия в равновесньх конечных элементах}

Основополагающие теоретические разработки метода равновесных конечных элементов представлены в [10], [13] и других работах. МКЭ в настоящей статье затронут только в контексте учета дисторсий для упругих и упругопластических систем.

Дискретная модель конструкции (рассматриваются только традиционные объекты строительной механики, а именно, стержневые системы и изгибаемые пластины) получается ее разделением на $\zeta$ конечных элементов. Составляющие вектора $\mathbf{F}$ обуславливаются распределенной элементной нагрузкой и нагрузкой линий стыка (сосредоточенные силы прихладываюотя $\mathbf{K}$ основным узлам элементов). Для стержневых систем принимается линейный закон распределения $\mathbf{S}_{e f}(x)$. Для изгибаемой пластины используется элемент второго порядка с квадратичным распределением усилий. Векторы обобщенных сил равновесного конечного элемента $\mathbf{P}_{k}$ и узловых усилий $\mathbf{S}_{e k}$ связаны уравнениями равновесия $[10,11,13]:$

$$
\mathbf{P}_{k}=\left[C_{k}\right] \mathbf{S}_{e, k}, \quad k=1,2, \ldots, \zeta .
$$

Объединить элементы в ансамбль можно, применяя матрицы $\left[B_{u}\right]$ и $\left[B_{c}\right]$. Эти матрипы определякт связь между перемещениями элементов

$$
\mathbf{u}_{e}^{*} \equiv\left\{\mathbf{u}_{e 1}^{*}, \mathbf{u}_{e 2}^{*}, \ldots, \mathbf{u}_{e k}^{*}, \ldots, \mathbf{u}_{e \zeta}^{*}\right\}^{T}
$$

и перемещениями дискретной модели конструкщии $\mathbf{u}_{e}$ и $\mathbf{u}_{c}$ :

$$
\mathbf{u}_{e}^{*}=\left[B_{u}\right] \mathbf{u}_{e}+\left[B_{c}\right] \mathbf{u}_{c} .
$$

Индекс $f$ для векторов $\mathbf{u}_{e}, \mathbf{S}_{e}$ и других здесь часто пропускается. С использованием результатов работы [13] уравнения равновесий (1), (6) и геометрические (2) уравнения записываются так:

$$
\begin{aligned}
& {\left[B_{u}\right]^{T} \mathbf{P}=\mathbf{F}, \text { или } \sum_{k=1}^{\zeta}\left[B_{u, k}\right]^{T} \mathbf{P}_{k}=\mathbf{F} ;} \\
& {\left[B_{c}\right]^{T} \mathbf{P}=\mathbf{R}_{e f}, \text { или } \sum_{k=1}^{\zeta}\left[B_{c, k}\right]^{T} \mathbf{P}_{k}=\mathbf{R}_{e f} ;} \\
& {\left[C_{k}\right]^{T} \mathbf{u}_{e k}^{*}=\left[D_{k}\right] \mathbf{s}_{e k}, \text { или }\left[A_{k}\right]^{T} \mathbf{u}_{e}=\left[D_{k}\right] \mathbf{s}_{e k} .}
\end{aligned}
$$


Здесь $\mathbf{P}-$ вектор обобщенных сил всех элементов: $\mathbf{P} \equiv\left\{\mathbf{P}_{1}, \mathbf{P}_{2}, \ldots, \mathbf{P}_{k}, \ldots, \mathbf{P}_{\zeta}\right\}^{T}$, $\left[B_{u, k}\right]^{T}\left[C_{k}\right]=\left[A_{k}\right]$, а матрица податливости $\left[D_{k}\right]$ определяется следующим образом:

$$
\left[D_{k}\right]=\iint_{A}\left[N_{s k}(\mathbf{x})\right]^{T}\left[d_{k}\right]\left[N_{s k}(\mathbf{x})\right] d A .
$$

Здесь $\left[N_{s k}(\mathbf{x})\right]-$ матрищы функщий формы элемента: $\mathbf{S}_{e k}(\mathbf{x})=\left[N_{s k}(\mathbf{x})\right] \mathbf{s}_{e k}$. Для получения (17) использовалась зависимость $\Theta_{e k}(\mathbf{x})=\left[\widetilde{d}_{k}\right] \mathbf{s}_{e k}(\mathbf{x})$, где $\left[\tilde{d}_{k}\right]=\left[d_{k}\right] d A-$ матрица податливости элемента $d A,\left[d_{k}\right]$ - матрица физических параметров. Нетрудно заметить, что

$$
\sum_{k=1}^{\zeta}\left[A_{k}\right] \mathbf{s}_{e f, k}=\mathbf{F} \quad \text { и }[A] \mathbf{S}_{e f}=\mathbf{F} .
$$

Используя (16), можно получпть традиционные в строительной механике уравнения для дискретной модели (2):

$$
[A]^{T} \mathbf{u}_{e f}=[D] \mathbf{s}_{e f},
$$

здесь $[D]=\left[\operatorname{diag}\left[D_{k}\right]\right], \quad k=1,2, \ldots, \zeta$. Так можно формировать систему уравнений (1), (2). Формула (17) может быть записана и так [18]:

$$
\left[D_{k}\right]=\int\left[N_{A}(\mathbf{x})\right]^{T}\left[N_{s k}(\mathbf{x})\right] d A\left[\operatorname{diag}\left[d_{k}\right]\right]=[V]\left[\operatorname{diag}\left[d_{k}\right]\right] .
$$

Здесь $\left[\operatorname{diag}\left[d_{k}\right]\right]$ - диагональная матрица, а матрища

$$
\left[V_{k}\right]=\iint_{A}\left[N_{s k}(\mathbf{x})\right]^{T}\left[N_{s k}(\mathbf{x})\right] d A
$$

Разрабатываются геометрические уравнения, когда дисторсия $\Theta_{q}(\mathbf{x})$ задана. В таком случае деформации элемента:

$$
\Theta_{e q, k}(\mathbf{x})=\left[\widetilde{d}_{k}\right] \mathbf{s}_{e q, k}(\mathbf{x})+\Theta_{q k}(\mathbf{x})=\left[\widetilde{d}_{k}\right] \mathbf{s}_{e q, k}(\mathbf{x})+\left[\widetilde{d}_{k}\right] \mathbf{s}_{q k}(\mathbf{x}) .
$$

Равенство виртуальных работ приводит к взаимосвязи перемещений ш $е \mathbf{c}$ деформациями элемента $\Theta_{e q, k}$ :

$$
\left[A_{k}\right]^{T} \mathbf{u}_{e q}=\left[D_{k}\right] \mathbf{s}_{e q, k}+\left[D_{k}\right] \mathbf{s}_{q k}
$$

Член $\left[D_{k}\right] \mathbf{s}_{q k}$, используя вектор деформаций $\Theta_{q k}$, может быть переписан следующим образом [16]:

$$
\left[D_{k}\right] \mathbf{s}_{q k}=\left[V_{k}\right]\left[\operatorname{diag}\left[d_{k}\right]\right] \mathbf{s}_{q k}=\left[V_{k}\right] \Theta_{q k} .
$$

Так получается равенство

$$
\left[A_{k}\right]^{T} \mathbf{u}_{e q}=\left[D_{k}\right] \mathbf{s}_{e q, k}+\left[V_{k}\right] \Theta_{q k}
$$


или

$$
\left[A_{k}\right]^{T} \mathbf{u}_{e q}=\left[D_{k}\right] \mathbf{S}_{e q, k}+\mathbf{q}_{k}
$$

Для всей конструкции уравнения (8) при наличии дисторсии:

$$
[A]^{T} \mathbf{u}_{e q}=[D] \mathbf{S}_{e q}+\mathbf{q} \cdot
$$

Как известно, практическая реализация метода конечных элементов основана на применении его матрицы жесткости. Одним из способов ее определения может быть следующий. Уравнения (16) представляются:

$$
\left[C_{k}\right]^{T}\left[B_{u, k}\right] \mathbf{u}_{e}=\left[D_{k}\right] \mathbf{S}_{e k}
$$

Решением систем (12), (20) определяется $\mathbf{P}_{k}$. Действуя оператором $\left[B_{u, k}\right]^{T}$ и произведя суммирование по $k$, огределяется

$$
\sum_{k=1}^{\zeta} \mathbf{F}_{k}=\sum_{k=1}^{\zeta}\left[B_{u, k}\right]^{T}\left[k_{k}\right]\left[B_{u, k}\right] \mathbf{u}_{e}, \quad \text { или }[k] \mathbf{u}_{e}=\mathbf{F} .
$$

Здесь матрица жесткости равновесного конечного элемента

$$
\left[k_{k}\right]=\left[C_{k}\right]\left[D_{k}\right]^{-1}\left[C_{k}\right]^{T}
$$

Имея в виду, что $\sum_{k=1}^{\zeta}\left[A_{k}\right]\left[D_{k}\right]^{-1} \mathbf{q}_{k}=\mathbf{F}_{q} \quad$ ( $\mathbf{F}_{q}-$ обусловленные дисторсией $\mathbf{q}$ силы $\left.\mathbf{F}_{q}=\sum_{k=1}^{\zeta} \mathbf{F}_{q, k}\right)$, можно с наименыними компьютерными ресурсами по схеме жесткостей конечных элементов сразу определить $\mathbf{u}_{e q}$ и $\mathbf{S}_{e q}$ (если за основу принимаются предлагаемые формулы (9),(10) [15]). Традиционная же схема жесткостей конечных элементов для определения, например $\mathbf{s}_{e q}$, требует дополнительного присуммирования к ним усилий $-[D]^{-1} \mathbf{q}$ (предварительного, в этом случае,' напряжения). Следует добавить, что от заданных пластических деформаций $\Theta_{p}^{*}$ вектор $\mathrm{F}_{q}$ определяется по той же. самой формуле $\sum_{k=1}^{\zeta}\left[A_{k}\right]\left[D_{k}\right]^{-1} \Theta_{p}^{*}=\mathbf{F}_{q} \quad$ [5]. Таким образом, и при наличии пшастических деформаций для болышого числа конечных элементов не требуется прямого формирования матриц $[\bar{H}]$ и $[\bar{G}]$, а тем более привлечения матрищ $[A]$ и $[D]$ для всей дискретной модели в целом. 


\section{4. Дисторсия при учете распределенной нагрузки}

При прямом учете нелинейного распределения усилий для стержневой конструхции увеличилось бы число переменных в разрешающих уравнениях. Распределенная нагрузка $p$ может быть сосредоточена в основных узлах элемента и в случае привлечения понятия дисторсии q (остается в силе линейное распределение усилий $\mathbf{S}_{e f}(x)$.

В системе уравнений (12) для $k$-го конечного элемента вводятся дисторсии $\mathbf{q}_{k}$, обусловленные распределенной нагрузкой $p$ :

$$
\mathbf{P}_{q, k}=\left[C_{k}\right]\left[D_{k}\right]^{-1} \mathbf{q}_{k} .
$$

Вектор узловых сил для дискретной модели:

$$
\mathbf{F}_{q}=\sum_{k=1}^{\zeta}\left[B_{u, k}\right]^{T} \mathbf{P}_{q, k}=\sum_{k=1}^{\zeta}\left[B_{u, k}\right]^{T}\left[C_{k}\right]\left[D_{k}\right]^{-1} \mathbf{q}_{k} \cdot
$$

Для матрицы $\left[C_{k}\right]$ по формуле (23) выделяются подматрицы $\left[C_{k}\right]$ и $\left[C_{k}^{\prime}\right]$ :

$$
\left[C_{k}\right]=\left[\begin{array}{l}
{\left[C_{k}\right]} \\
{\left[C_{k}^{\prime}\right]}
\end{array}\right]
$$

Для закрепленного элемента выделение подматрицы $\left[C_{k}\right]$ эквивалентно подбору основной системы, для которой и была построена матрица $\left[D_{k}\right]$. Так определяются степени свободы элемента, как абсолютно жесткого. Подматрице [C,k] отвечают ненулевые составляющие $\mathbf{P}_{c q, k}^{\prime \prime}$ вектора обобщенных сил $\mathbf{P}_{c q, k}$ абсолютно жесткого элемента при действии $p$ :

$$
\mathbf{P}_{c q, k} \equiv\left\{\begin{array}{l}
\mathbf{P}_{c q, k}^{\prime} \\
\mathbf{P}_{c q, k}^{\prime \prime}
\end{array}\right\} \equiv\left\{\begin{array}{l}
\mathbf{0} \\
\mathbf{P}_{c q, k}^{\prime \prime}
\end{array}\right\} .
$$

Составляющими вектора $\mathbf{P}_{c q, k}^{\prime \prime}$ являются опорные реакции в статически определимой системе, взятые со знаком минус. Вектор узловых сил $\mathbf{F}_{c q}$ рассчитывается по формуле

$$
\mathbf{F}_{c q}=\sum_{k=1}^{\zeta}\left[B_{u, k}\right]^{T} \mathbf{P}_{c q, k}
$$

Тогда вектор $\mathbf{F}=\mathbf{F}_{c q}$ (при наличии и других внешгии нагрузок $\mathbf{F}$ представляет их сумму). Следовательно, при действии $p$ для формул (11) вводятся векторы $\mathbf{F}_{c q}$ и $\mathbf{q}$ (матричная форма метода перемещений в строительной механике стержневых систем [14]).

От выбора подматрицы $\left[C_{k}\right]$ (от выбора статически определимой системы) дополнительная энергия не зависит. Это учитывалось в математических моделях 
оценки остаточных перемещений упругопластических систем при действии повторных нагружений [19].

\section{5. Экстремальные задачи для упругой конструкпии}

Зависимости (11) являются решением системы уравнений Эйлера-Лагранжа (1) - (3), (8) для упругой системы при наличии нагрузки $\mathbf{F}$ и дисторсии q . Усилия, перемещения и деформации могут быть определены и решением двойственной пары задач математического программирования. Первая из этих задач построена на основе принципа минимума дополнительной энергии $[2,4,16]$ :

найти

$$
\min \left\{\frac{1}{2} \mathbf{S}_{e}^{T}[D] \mathbf{S}_{e}+\mathbf{S}_{e}^{T} \mathbf{q}\right\}
$$

при условиях

$$
[A] \mathbf{S}_{e}=\mathbf{F} .
$$

Вторая задача формулируется с привлечением принципа минимума полной потенциальной энергии:

найти

$$
\min \left\{\frac{1}{2} \mathbf{S}_{e}^{T}[D] \mathbf{S}_{e}-\mathbf{F}^{T} \mathbf{u}_{e}\right\}
$$

при условиях

$$
[A]^{T} \mathbf{u}_{e}=[D] \mathbf{S}_{e}+\mathbf{q} .
$$

Уравнения (28), (30) представляют собой обобщенную задачу Лагранжа (1) - (3), (8).

При наличии только дисторсии q получается следующая пара задач: найти

$$
\min \left\{\frac{1}{2} \mathbf{S}_{e q}^{T}[D] \mathbf{S}_{e q}+\mathbf{S}_{e q}^{T} \mathbf{q}\right\}
$$

при условиях

$$
[A] \mathbf{S}_{e q}=\mathbf{0}
$$

найти

$$
\min \frac{1}{2} \mathbf{S}_{e q}^{T}[D] \mathbf{S}_{e q}
$$

при условиях

$$
[A]^{T} \mathbf{u}_{e q}=[D] \mathbf{S}_{e q}+\mathbf{q}
$$


Условия-ограничения (32), (34), записанные вместе, являются обобщенной задачей Лагранжа (8). Каждая из представленных задач математического программирования может быть сведена к проблеме безусловной минимизации.

\section{6. Экстремальные задачи для упругопластической конструкции}

6. 1. Дисторсия в упругопластической стержневой системе

В работах $[4,6,7]$ представлены экстремальные энерцетические принципы для определения деформаций $\Theta_{p}$ идеально упругопластической конструкции при заданном только векторе внешних нагрузок F. Здесь они применяются и для заданных дисторсий $\mathbf{q}=\Theta_{q t}+\Theta_{q e}+\Theta_{q c}$, когда возможная разгрузка не учитывается. Пластические деформации локализируются в узлах стержневого элемента (принимается конщепция идеального пластического шарнира).

Статически допустимый самоуравновешенный вектор усилий $\mathbf{S}_{r q}=\mathbf{S}_{r}+\mathbf{S}_{e q}$ удовлетворяет уравнениям равновесия

$$
[A] \mathbf{S}_{r q}=\mathbf{0}
$$

и условиям текучести

$$
[\Phi]\left(\mathbf{S}_{e f}+\mathbf{S}_{r q}\right) \leq \mathbf{S}_{0}
$$

Здесь $\mathbf{S}_{e f}=[\alpha] \mathbf{F}, \mathbf{S}_{0}$ - заданные векторы квазиупругих и предельных усилий (в пределах каждого из элементов $\mathbf{s}_{0 \zeta}=$ const ).

В идеально упругопластической системе до достижения ее пластического разрушения из всех статически допустимых самоуравновешенных векторов усилий $\mathbf{S}_{r q}$ действительным является тот, при котором дополнительная энереия минимальна.

Этому принципу отвечает следующая экстремальная задача:

найти

$$
\min \left\{\frac{1}{2} \mathbf{S}_{r q}^{T}[D] \mathbf{S}_{r q}+\mathbf{S}_{r q}^{T} \mathbf{q}\right\}+\mathrm{C}_{1}
$$

при условиях

$$
\begin{aligned}
& {[A] \mathbf{S}_{r q}=\mathbf{0},} \\
& {[\Phi]\left(\mathbf{S}_{e f}+\mathbf{S}_{r q}\right) \leq \mathbf{S}_{0} .}
\end{aligned}
$$

Здесь константа $\mathrm{C}_{1}=\frac{1}{2} \mathbf{S}_{e q}^{T}[D] \mathbf{S}_{e q}$. В качестве неизвестного здесь выступает вектор самоуравновешенных усилий $\mathbf{s}_{r q}$. Двойственной к задаче $(35)-(36)$ является следующая задача (если функцию цели умножить на -1 ): 
найти

$$
\min \left\{\frac{1}{2} \mathbf{S}_{r q}^{T}[D] \mathbf{S}_{r q}+\mathbf{S}_{r q}^{T}[\Phi]^{T} \lambda+\lambda^{T}\left(\mathbf{S}_{0}-[\Phi]\left(\mathbf{S}_{e f}+\mathbf{S}_{r q}\right)\right)\right\}-\mathrm{C}_{1}
$$

при условиях

$$
\begin{aligned}
& {[A]^{T} \mathbf{u}_{r q}=[D] \mathbf{S}_{r q}+\mathbf{q}+[\Phi]^{T} \lambda,} \\
& \lambda \geq \mathbf{0} .
\end{aligned}
$$

В качестве неизвестных здесь выступают векторы $\mathbf{S}_{r q}, \lambda \mathbf{u}_{r q}$. Уравнения (38) вместе с ограничением (39) $\lambda \geq 0$ определяют, как известно, кинематически допустимый вектор перемещений $\mathbf{u}_{r q}$. Экстремальнал задача (37)-(39) отвечает энергетическому принципу минимума полной потенциальной энергии для кинематически допустимых перемещений $\mathbf{u}_{r q}$.

Уравнения (38) показывают, что только лишь дисторсия q и пластические деформации $\Theta_{p}=[\Phi]^{T} \lambda$ не удовлетворяют уравнениям геометрической совместности деформаций. Это их объединяющее свойство и было использовано при обосновании применения формул (9), (10) в анализе НДС угругих и упругопластических конструкций. С другой стороны, обобщенная задача Лагранжа такова:

$$
\begin{aligned}
& {[A] \mathbf{S}_{r q}=\mathbf{0},} \\
& {[\Phi]\left(\mathbf{s}_{e f}+\mathbf{S}_{r q}\right) \leq \mathbf{S}_{0},} \\
& {[A]^{T} \mathbf{u}_{r q}=[D] \mathbf{s}_{r q}+\mathbf{q}+[\Phi]^{T} \lambda,} \\
& \lambda \geq \mathbf{0}, \quad \lambda^{T}\left(\mathbf{s}_{0}-[\Phi]\left(\mathbf{s}_{e f}+\mathbf{S}_{r q}\right)\right)=0 .
\end{aligned}
$$

Для определения переменных $\mathbf{u}_{r q}, \mathbf{s}_{r q}$, происходящих от дисторсий и пластических деформаций, могут использоваться те же самые матричные зависимости методов конечных разностей или конечных элементов, разработанные для упругих систем:

$$
\begin{gathered}
\mathbf{u}_{r q}=\left([A][D]^{-1}[A]^{T}\right)^{-1}[A][D]^{-1}\left(\mathbf{q}+[\Phi]^{T} \lambda\right)=[\alpha]^{T}\left(\mathbf{q}+[\Phi]^{T} \lambda\right)=[\bar{H}]\left(\mathbf{q}+[\Phi]^{T} \lambda\right), \\
\mathbf{s}_{r q}=\left\{[D]^{-1}[A]^{T}\left([A][D]^{-1}[A]^{T}\right)^{-1}[A][D]^{-1}-[D]^{-1}\right\}\left(\mathbf{q}+[\Phi]^{T} \lambda\right)= \\
=\left\{[\alpha][A][D]^{-1}-[D]^{-1}\right\}\left(\mathbf{q}+[\Phi]^{T} \lambda\right)=[\bar{G}]\left(\mathbf{q}+[\Phi]^{T} \lambda\right), \\
{[\Phi]\left(\mathbf{s}_{e f}+[\bar{G}]\left(\mathbf{q}+[\Phi]^{T} \lambda\right)\right) \leq \mathbf{s}_{0},} \\
\lambda \geq \mathbf{0}, \quad \lambda^{T}\left(\mathbf{s}_{0}-[\Phi]\left(\mathbf{s}_{e f}+[\bar{G}]\left(\mathbf{q}+[\Phi]^{T} \lambda\right)\right)\right)=0 .
\end{gathered}
$$


Следовательно, понятие дисторсии как несовместной деформации выступает связующим звеном для задач анализа в упругой и упругопластической стадиях работы системы:

$$
[B] \mathbf{q} \neq \mathbf{0}, \quad[B][\Phi]^{T} \lambda \neq \mathbf{0} .
$$

Математические модели задач (35)-(36), (37)-(39) удобно использовать в шаговых алгоритмах решения задач анализа упрутопластических конструхций.

Решение задачи (37)-(39) упрощается, если использовать уравнения совместности

$$
[B] \Theta_{r q}=\mathbf{0}
$$

для деформаций $[5,19] \Theta_{r q}=[D] \mathbf{S}_{r q}+\mathbf{q}+[\Phi]^{T} \lambda$ :

$$
\left[B_{\lambda}\right] \lambda-\left[B_{r}\right] \mathbf{S}_{r q}=-[B] \mathbf{q} \text {. }
$$

Они могут быть получены и из уравнений (38) исключением перемещений $\mathbf{u}_{r q}$. Здесь

$$
[B]=\left[\left[A^{\prime \prime}\right]^{T}\left(\left[A^{\prime}\right]^{T}\right)^{-1},-[\mathrm{I}]\right] \text { и }\left[B_{r}\right]=-\left[A^{\prime \prime}\right]^{T}\left(\left[A^{\prime}\right]^{T}\right)^{-1}\left[D^{\prime}\right]+\left[D^{\prime \prime}\right]
$$

Матрица $\left[B_{\lambda}\right]=[B][\Phi]^{T}$. Тогда математическая модель (37)-(39) может быть записана следующим образом:

найти

$$
\min \left\{\frac{1}{2} \mathbf{S}_{r q}^{T}[D] \mathbf{S}_{r q}+\mathbf{S}_{r q}^{T}[\Phi]^{T} \lambda+\lambda^{T}\left(\mathbf{S}_{0}-[\Phi]\left(\mathbf{S}_{e f}+\mathbf{S}_{r q}\right)\right)\right\}-\mathrm{C}_{1}
$$

при условиях

$$
\left[B_{\lambda}\right] \lambda-\left[B_{r}\right] \mathbf{S}_{r q}=-[B] \mathbf{q}
$$

$$
\lambda \geq \mathbf{0} \text {. }
$$

При оптимальном решении задачи (41)-(43) $\mathbf{s}_{r q}^{*}, \lambda^{*}$ последний член функции цели (41) равен нулю:

$$
\lambda^{* T}\left(\mathbf{S}_{0}-[\Phi]\left(\mathbf{S}_{e f}+\mathbf{S}_{r q}^{*}\right)\right)=0
$$

Это относится и к оптимальному решению задачи $(37)-(39) \mathbf{s}_{r q}^{*}, \lambda^{*}, \mathbf{u}_{r q}^{*}$.

Вводом остаточных усилий $\quad \mathbf{S}_{r}=\mathbf{S}_{r q}-\mathbf{S}_{e q} \quad\left(\mathbf{S}_{e q}=[\bar{G}] \mathbf{q} \quad\right), \quad$ перемещений $\mathbf{u}_{r}=\mathbf{u}_{r q}-\mathbf{u}_{e q}$ математические модели (35) - (36), (37) - (39) принимает вид: найти

$$
\min \left\{\frac{1}{2} \mathbf{S}_{r}^{T}[D] \mathbf{S}_{r}\right\}
$$


при условиях

$$
\begin{aligned}
& {[A] \mathbf{S}_{r}=\mathbf{0},} \\
& {[\Phi]\left(\mathbf{S}_{e f}+\mathbf{S}_{e q}+\mathbf{S}_{r}\right) \leq \mathbf{S}_{0} .}
\end{aligned}
$$

Решением задачи (45)-(46) определяются остаточные усилия $\mathbf{s}_{r}^{*}$. Для математической модели (37) - (39) :

найти

$$
\min \left\{\frac{1}{2} \mathbf{S}_{r}^{T}[D] \mathbf{S}_{r}+\mathbf{S}_{r}^{T}[\Phi]^{T} \lambda+\lambda^{T}\left(\mathbf{S}_{0}-[\Phi]\left(\mathbf{S}_{e f}+\mathbf{S}_{e q}+\mathbf{S}_{r}\right)\right)\right\}
$$

при условиях

$$
\begin{aligned}
& {[A]^{T} \mathbf{u}_{r}=[D] \mathbf{S}_{r}+[\Phi]^{T} \lambda,} \\
& \lambda \geq \mathbf{0} .
\end{aligned}
$$

Неизвестными здесь выступают $\mathbf{S}_{r}, \mathbf{u}_{r}, \lambda$. Деформации $\Theta_{r q}$ определяются с учетом (48): $\Theta_{r q}=[D] \mathbf{S}_{r q}+\mathbf{q}+[\Phi]^{T} \lambda$. Если $\mathbf{q}=\mathbf{0}$, математические модели (45)-(46), (47)-(49), учитывающие только F , приобретают известный по работе [7] вид : найти

$$
\min \left\{\frac{1}{2} \mathbf{S}_{r}^{T}[D] \mathbf{S}_{r}\right\}
$$

при условиях

$$
\begin{aligned}
& {[A] \mathbf{S}_{r}=\mathbf{0},} \\
& {[\Phi]\left(\mathbf{S}_{e f}+\mathbf{S}_{r}\right) \leq \mathbf{S}_{0} .}
\end{aligned}
$$

В качестве неизвестных здесь выступают остаточные усилия $\mathbf{s}_{r}$. Двойственная $\mathbf{k}$ задаче (50)-(51) задача такова:

найти

$$
\min \left\{\frac{1}{2} \mathbf{S}_{r}^{T}[D] \mathbf{S}_{r}+\mathbf{S}_{r}^{T}[\Phi]^{T} \lambda+\lambda^{T}\left(\mathbf{S}_{0}-[\Phi]\left(\mathbf{S}_{e f}+\mathbf{S}_{r}\right)\right)\right\}
$$

при условиях

$$
\begin{aligned}
& {[A]^{T} \mathbf{u}_{r}=[D] \mathbf{S}_{r}+[\Phi]^{T} \lambda,} \\
& \lambda \geq \mathbf{0} .
\end{aligned}
$$

Отределяются векторы $\mathbf{s}_{r}^{*}, \lambda^{*}, \mathbf{u}_{r}^{*}$. Тогда задача Лагранжа:

$$
\begin{aligned}
& {[A] \mathbf{S}_{r}=\mathbf{0},} \\
& {[\Phi]\left(\mathbf{S}_{e f}+\mathbf{S}_{r}\right) \leq \mathbf{S}_{0},} \\
& {[A]^{T} \mathbf{u}_{r}=[D] \mathbf{S}_{r}+[\Phi]^{T} \lambda,} \\
& \lambda \geq \mathbf{0}, \quad \lambda^{T}\left(\mathbf{S}_{0}-[\Phi]\left(\mathbf{S}_{e f}+\mathbf{S}_{r}\right)\right)=0 .
\end{aligned}
$$


Исключением остаточных перемещений $\mathbf{u}_{r}$ и остаточных усилий $\mathbf{S}_{r}$ :

$$
\mathbf{u}_{r}=[\bar{H}][\Phi]^{T} \lambda=[H] \lambda, \quad \mathbf{S}_{r}=[\bar{G}][\Phi]^{T} \lambda=[G] \lambda
$$

система (55) упроцается:

$$
\begin{aligned}
& \lambda \geq \mathbf{0}, \\
& \mathbf{s}_{0}-[\Phi]\left(\mathbf{S}_{e f}+[G] \lambda\right) \geq \mathbf{0}, \\
& \lambda^{T}\left(\mathbf{S}_{0}-[\Phi]\left(\mathbf{S}_{e f}+[G] \lambda\right)\right)=0 .
\end{aligned}
$$

Решгением системы (57) является $\lambda^{*}$, а согласно формулам (56) - и $\mathbf{u}_{r}^{*}=[H] \lambda^{*}$, $\mathbf{S}_{r}^{*}=[G] \lambda^{*}$. Формулы (56) эквивалентны формулам (9)-(10), когда дисторсия q заменяется известными пластическими деформациями $\Theta_{p}^{*}=[\Phi]^{T} \lambda^{*}$.

Далее рассматривается математическая модель (52) - (54), когда известен вектор $\lambda^{*}$, т.е. вектор $\Theta_{p}^{*}=[\Phi]^{T} \lambda^{*}$. Тогда

$$
\lambda^{* T}[\Phi] \mathbf{S}_{r}=\lambda^{* T}\left(\mathbf{S}_{0}-[\Phi] \mathbf{s}_{e}\right)=\text { const }
$$

При обозначении этой константы через $\mathrm{C}_{2}$ задача (52) - (54) представляется так: найти

$$
\min \frac{1}{2} \mathbf{s}_{r}^{T}[D] \mathbf{s}_{r}+C_{2}
$$

при условиях

$$
[A]^{T} \mathbf{u}_{r}=[D] \mathbf{S}_{r}+\Theta_{p}^{*},
$$

Определяются векторы $\mathbf{u}_{r}^{*}$, и $\mathbf{S}_{r}^{*}$. Математическая модель (59)-(60) отвечает одной из двойственных формулировок минимального принципа Койтера для остаточных напряжений [6]. В уравнениях (60) заданные пластические деформации $\Theta_{p}^{*}$ учитываются с привлечением остаточных, а не суммарных усилий и перемещений. С другой стороны, считая, что $\mathbf{q}=[\Phi]^{T} \lambda^{*}$, для подсчета остаточных усилий $\mathbf{S}_{e q}^{*}=\mathbf{S}_{r}^{*}$ и перемещений $\mathbf{u}_{e q}^{*}=\mathbf{u}_{r}^{*}$ можно использовать математические модели задач (31)(32), (33)-(34), подразумевая квазиупругость конструкции. В этом случае условия текучести

$$
[\Phi] \mathbf{S}_{r}^{*} \leq \mathbf{S}_{0}
$$


всегда будут удовлетворены.

С учетом уравнений (40) для остаточных деформаций

$$
\left[B_{r}\right] \mathbf{S}_{r}=\left[B_{\lambda}\right] \lambda^{*}
$$

математическая модель (59)-(60) принимает вид:

найти

$$
\min \frac{1}{2} \mathbf{S}_{r}^{T}[D] \mathbf{S}_{r}+C_{2}
$$

при условиях

$$
\left[B_{r}\right] \mathbf{S}_{r}=\left[B_{\lambda}\right] \lambda^{*}
$$

Заданным здесь является вектор $\lambda^{*}$. Определяется оптимальное решение $\mathbf{s}_{r}^{*}$.

\section{2. Дисторсия в упругопластических изгибаемых пластинах}

В $i$-ом узле $k$-го конечного элемента $(k=1,2, \ldots, \zeta)$ изгибаемой пластины действуют изгибающие моменты [15]

$$
\mathbf{M}_{e f, k, i} \equiv\left\{M_{11, k, i}, M_{22, k, i}, M_{12, k, i}\right\}^{T} .
$$

Уравнения равновесия дискретной модели $[A] \mathbf{M}_{e f}=\mathbf{F}$ (для остаточных моментов $\left.[A] \mathbf{M}_{r}=\mathbf{0}\right)$. Деформации элемента представляются так:

$$
\Theta_{e f, k}(\mathbf{x})=\left[\widetilde{d}_{k}\right] \mathbf{M}_{e f, k}(\mathbf{x}) \text {. }
$$

Здесь $\left[\tilde{d}_{k}\right] \quad-$ матрица податливости элемента $d A$. Уравнения, аналогичные уравнениям (16), для конечного элемента и всей дискретной модели пластины таковы:

$$
\left[A_{k}\right]^{T} \mathbf{u}_{e f}=\left[D_{k}\right] \mathbf{M}_{e f, k}, \quad k=1,2, \ldots, \zeta ;[A]^{T} \mathbf{u}_{e f}=[D] \mathbf{M}_{e f} .
$$

Далее записываются геометрические уравнения элемента при наличии дисторсии $\Theta_{q}(\mathbf{x})$ и пластических деформаций $\Theta_{p}(\mathbf{x}) ; \Theta_{p k}(\mathbf{x})=\left[N_{p k}(\mathbf{x})\right] \Theta_{p k}$. Тогда

$$
\Theta_{r q, k}(\mathbf{x})=\left[\widetilde{d}_{k}\right] \mathbf{M}_{r q, k}(\mathbf{x})+\Theta_{q k}(\mathbf{x})+\Theta_{p k}(\mathbf{x})
$$

и уравнения $k$-го элемента

$$
\left[A_{k}\right]^{T} \mathbf{u}_{r q}=\left[D_{k}\right] \mathbf{M}_{r q, k}+\left[V_{k}\right] \Theta_{q k}+\left[V_{p k}\right] \Theta_{p k} .
$$

Здесь $\left[V_{p k}\right]=\int_{A_{k}}\left[N_{m k}(\mathbf{x})\right]^{T}\left[N_{p k}(\mathbf{x})\right] d A . \quad\left[V_{k}\right]=\left[V_{p k}\right]$, если $\left[N_{m k}(\mathbf{x})\right]=\left[N_{p k}(\mathbf{x})\right]$. 
Обозначаем $\left[V_{k}\right] \Theta_{q k}=\mathbf{q}_{k}\left(\left[D_{k}\right] \mathbf{M}_{q k}=\left[V_{k}\right]\left[\operatorname{diag}\left[d_{k}\right]\right] \mathbf{M}_{q k}=\left[V_{k}\right] \Theta_{q k}=\mathbf{q}_{k}\right)$. Тогда формула (63) принимает вид:

$$
\left[A_{k}\right]^{T} \mathbf{u}_{r q}=\left[D_{k}\right] \mathbf{M}_{r q, k}+\mathbf{q}_{k}+\Theta_{p k} \quad \text { и }[A]^{T} \mathbf{u}_{r q}=[D] \mathbf{M}_{r q}+\mathbf{q}+\Theta_{p}
$$

Вектор $\Theta_{p k}(\mathbf{x})$ связан с вектором пластических кривизн: $\Theta_{p k}(\mathbf{x})=\left[N_{p k}(\mathbf{x})\right] d A \mathbf{K}_{p k}$. Для проверяемых в узловых точках элемента условий текучести Мизеса пластические кривизны $\mathbf{K}_{p k, i}$ представляются следующим образом:

$$
\mathbf{K}_{p k, i}=2 \lambda_{k, i}\left[\varphi_{k}\right] \mathbf{M}_{k, i}, \quad \mathbf{M}_{k, i}=\mathbf{M}_{r q, k, i}+\mathbf{M}_{e f, k, i} .
$$

Здесь $\left[\varphi_{k}\right]$ - матрица Мизеса. Для конечного элемента в целом

$$
\mathbf{K}_{p k}=2\left[\operatorname{diag}\left[\boldsymbol{\varphi}_{k}\right]\right]\left[\Gamma_{k}\left(\mathbf{M}_{k, i}\right)\right] \lambda_{k} .
$$

Структура матрицы $\left[\Gamma_{k}\right]$ для конечного элемента:

$$
\left[\Gamma_{k}\right]=\left[\operatorname{diag}\left[\left(\mathbf{M}_{r q, k, i}+\mathbf{M}_{e f, k, i}\right)\right]\right], i=1,2, \ldots, s .
$$

В случае интегрального условия текучести уравнения (63) для элемента принимают вид:

$$
\left[A_{k}\right]^{T} \mathbf{u}_{r q}=\left[D_{k}\right] \mathbf{M}_{r q, k}+\mathbf{q}_{k}+2\left[V_{k}\right]\left[\Pi_{k}\right] \mathbf{M}_{k} \lambda_{k} .
$$

Так выявляется матрица интегрального условия текучести $\left[\Phi_{k}\right]$ :

$$
\left[\Phi_{k}\right]=\left[V_{k}\right]\left[\Pi_{k}\right],\left[\Pi_{k}\right]=\left[\operatorname{diag}\left[\varphi_{k}\right]\right] .
$$

С учетом условий текучести Мизеса математические модели нелинейных задач анализа пластины строятся аналогично математическим моделям задач (35)-(36), (37)-(39):

найти

$$
\min \left\{\frac{1}{2} \mathbf{M}_{r q}^{T}[D] \mathbf{M}_{r q}+\mathbf{M}_{r q}^{T} \mathbf{q}\right\}
$$

при условиях

$$
\begin{aligned}
& {[A] \mathbf{M}_{r q}=\mathbf{0},} \\
& {\left[\Gamma_{k}\left(\mathbf{M}_{r q, k, i}+\mathbf{M}_{e f, k, i}\right)\right]^{T}\left[\Pi_{k}\right]\left(\mathbf{M}_{r q, k, i}+\mathbf{M}_{e f, k, i}\right) \leq \mathbf{C}_{k}}
\end{aligned}
$$

для всех $k=1,2, \ldots, \zeta, i=1,2, \ldots, s$.

Здесь $\mathbf{M}_{e f}=[\propto] \mathbf{F}-$ вектор моментов упругого расчета, $\mathbf{C}_{k}-$ вектор пластических констант $\left(C_{i} \equiv\left(M_{0 i}\right)^{2}\right)$. Определяется $\mathbf{M}^{*}{ }_{r q}$.

Кинематическая формулировка задачи (она записана с привлечением матриц для всей дискретной модели конструкции): 
найти

$$
\begin{aligned}
& \min \left\{\frac{1}{2} \mathbf{M}_{r q}^{T}[D] \mathbf{M}_{r q}+2 \lambda^{T}\left[\Gamma\left(\mathbf{M}_{r q}+\mathbf{M}_{e f}\right)\right]^{T}[\Pi] \mathbf{M}_{r q}+\right. \\
& \left.+\lambda^{T}\left\{\mathbf{C}-\left[\Gamma\left(\mathbf{M}_{r q}+\mathbf{M}_{e f}\right)\right]^{T}[\Pi]\left(\mathbf{M}_{r q}+\mathbf{M}_{e f}\right)\right\}\right\}
\end{aligned}
$$

при условиях:

$$
[A]^{T} \mathbf{u}_{r q}=[D] \mathbf{M}_{r q}+\mathbf{q}+2[\Pi]\left[\Gamma\left(\mathbf{M}_{r q}+\mathbf{M}_{e f}\right)\right] \lambda, \quad \lambda \geq \mathbf{0} .
$$

Константа $\mathrm{C}_{1}$ в статической и кинематической формулировках задач не приводится. Решением задачи (66)-(67) определяются $\quad \mathbf{u}^{*}{ }_{r q}, \mathbf{M}_{r q}^{*}, \lambda^{*}$. Дальнейшее совершенствование математических моделей задач (64)-(65), (66)-(67) должно быть связано с учетом энергии диссипации в местах соединения конечных элементов. Математические модели задач нетрудно прребразовать для метода конечных разностей.

\section{3 О задаче анализа приспосабливающейся конструкпии}

Рассматривается НДС упругопластической приспосабливающейся конструкции, близкое к ее циклически-пластическому разрушению (коэффициент запаса по приспособляемости близок $\mathrm{k}$ 1). В этом случае для всех историй нагружения существует единственный вектор остаточных усилий $\mathbf{s}_{r}^{*}$. Пусть внешгняя нагрузка $\mathbf{F}(t)$ задана пределами своего изменения $\mathbf{F}_{\text {sup }} \leq \mathbf{F}(t) \leq \mathbf{F}_{\text {inf }}$, а дисторсия $\mathbf{q}=$ const. Экстремальные усилия упругого расчета $\mathbf{S}_{e f, j, \max }, \mathbf{S}_{e f, j, \min }$ определяются $\mathbf{c}$ привлечением матрищы влияния $[\alpha]$ и являются линейными функапиями от $\mathbf{F}_{\text {sup }}, \mathbf{F}_{i n f}$ (для определения $\mathbf{S}_{e f, j, \max }, \mathbf{S}_{e f, j, \min }$ можно непосредственно использовать и алторитмы МКЭ). Векторы $\mathbf{S}_{e f, j, \max }, \mathbf{S}_{e f, j, \min }$ представляют собой все вершины тодографа упругих усилий $\mathrm{S}_{e f}(t)$ (через $j$ определяется симметричная пара, их множество ). Самоуравновешенные усилия от дисторсии q рассчитываются согласно формуле $\mathbf{S}_{e q}=[\bar{G}] \mathbf{q}$. Окончательно экстремальные усилия упругого расчета выглядят так:

$$
\mathbf{S}_{e f, \max }=\mathbf{S}_{e f, j, \max }+\mathbf{S}_{e q}, \quad \mathbf{S}_{e j, \min }=\mathbf{S}_{e f, j, \min }+\mathbf{S}_{e q} .
$$

Формированием новой матрицы влияния $[[\alpha],[\bar{G}]]$ можно учесть повторнопеременный характер изменения $\mathbf{q}$ (для подсчета векторов $\mathbf{S}_{e j, \max }, \mathbf{S}_{e j, \min }$ ). 
Нелинейная математическая модель задачи анализа приспосабливающейся конструкции в статической формулировке такова:

найти

$$
\min \frac{1}{2} \mathbf{s}_{r}^{T}[D] \mathbf{S}_{r}
$$

при условиях

$$
[A] \mathbf{S}_{r}=\mathbf{0}, \quad \mathbf{f}\left(\mathbf{S}_{r}+\mathbf{S}_{e j, \text { max }}\right) \leq \mathbf{C}, \mathbf{f}\left(\mathbf{S}_{r}+\mathbf{S}_{e j, \min }\right) \leq \mathbf{C} \text { для всех } j \in J .(69)
$$

Определяется вектор $\mathbf{S}_{r}^{*}$.

Кинематическая формулировка задачи:

найти

$$
\begin{aligned}
& \min \left\{0.5 \cdot \mathbf{S}_{r}^{T}[D] \mathbf{S}_{r}+\sum_{j} \lambda_{j, \max }^{T}\left[\nabla \mathbf{f}\left(\mathbf{S}_{r}+\mathbf{S}_{e j, \max }\right)\right] \mathbf{S}_{r}+\right. \\
& +\sum_{j} \lambda_{j, \min }^{T}\left[\nabla \mathbf{f}\left(\mathbf{S}_{r}+\mathbf{S}_{e j, \min }\right)\right] \mathbf{S}_{r}+\sum_{j} \lambda_{j, \max }^{T}\left[\mathbf{C}-\mathbf{f}\left(\mathbf{S}_{r}+\mathbf{S}_{e j, \max }\right)\right]+ \\
& \left.+\sum_{j} \lambda_{j, \min }^{T}\left[\mathbf{C}-\mathbf{f}\left(\mathbf{S}_{r}+\mathbf{S}_{e j, \min }\right)\right]\right\}
\end{aligned}
$$

при условиях

$$
\begin{aligned}
& {[D] \mathbf{S}_{r}+\sum_{j}\left[\nabla \mathbf{f}\left(\mathbf{S}_{r}+\mathbf{S}_{e j, \max }\right)\right]^{T} \lambda_{j, \max }+} \\
& +\sum_{j}\left[\nabla \mathbf{f}\left(\mathbf{S}_{r}+\mathbf{S}_{e j, \min }\right)\right]^{T} \lambda_{j, \min }-[A]^{T} \mathbf{u}_{r}=\mathbf{0}, \text { дाI всех } j \in J . \\
& \lambda_{j, \max } \geq \mathbf{0}, \quad \lambda_{j, \min } \geq \mathbf{0} .
\end{aligned}
$$

Здесь $[\nabla \mathbf{f}]$ - матрица градиентов нелинейных условий текучести. Условия являются геометрическими уравнениями $[A]^{T} \mathbf{u}_{r}=\Theta_{r}$. Решением задачи (70), (71), определяются $\mathbf{S}_{r}^{*}, \mathbf{u}_{r}^{*}, \lambda_{j, \max }^{*}, \lambda_{j, \min }^{*}$. Математические модели задач (68) - (69), (70) - (71) можно записать в виде: найти $\min \left\{\frac{1}{2} \mathbf{S}_{r q}^{T}[D] \mathbf{S}_{r q}+\mathbf{S}_{r q}^{T} \mathbf{q}\right\} \quad$ при условиях (69) (в них вводятся $\mathbf{S}_{r q}, \mathbf{S}_{e f, \max }, \mathbf{S}_{e f, \min }$ ); найти $\min$ (70) (вводятся $\mathbf{S}_{r q}$, $\mathbf{S}_{e f, j, \max }, \mathbf{S}_{e f, j, \min }$, ) при условиях

$$
\begin{aligned}
& {[D] \mathbf{S}_{r q}+\mathbf{q}+\sum_{j}\left[\nabla \mathbf{f}\left(\mathbf{S}_{r q}+\mathbf{S}_{e f, j, \max }\right)\right]^{T} \lambda_{j, \max }+} \\
& +\sum_{j}\left[\nabla \mathbf{f}\left(\mathbf{S}_{r q}+\mathbf{S}_{e f, j, \min }\right)\right]^{T} \lambda_{j, \min }-[A]^{T} \mathbf{u}_{r q}=\mathbf{0}, \text { для всех } j \in J . \\
& \lambda_{j, \max } \geq \mathbf{0}, \lambda_{j, \min } \geq \mathbf{0} .
\end{aligned}
$$




\section{7. Пример расчета упругопластической пластины}

Методом конечных разностей определяются прогибы упругопластической пластины (рис.1) для нагрузки, близкой $\mathrm{k}$ предельной: распределенной $\mathrm{p}=1.1126 M_{0} / \Delta^{2}=17.816 M_{0} L^{-2} \quad$ (шаг $\Delta=0.25 \mathrm{~L}$ ), и скопившихся пластических деформаций

$\Theta_{p} \equiv\left\{\Theta_{11,1}, \Theta_{22,1}, \Theta_{11,2}, \Theta_{22,2}, \Theta_{11,3}, \Theta_{22,3}, \Theta_{11,4}, \Theta_{22,4}, \Theta_{11,5}, \Theta_{22,5} \Theta_{12,5}, \Theta_{11,6}\right.$, $\left.\Theta_{22,6}, \Theta_{12,6}, \Theta_{12,7}, \Theta_{12,8}, \Theta_{12,9}, \Theta_{12,10}\right\}^{T} \equiv\{0.1568,0.5083,0.2034,0.6915,0,0,0,0$, $0.0313,0.0707,-0.1223,0.0825,0.1637,-0.4609,-0.0933,0,-0.2433,-0.2998\}^{\mathrm{T}}$.

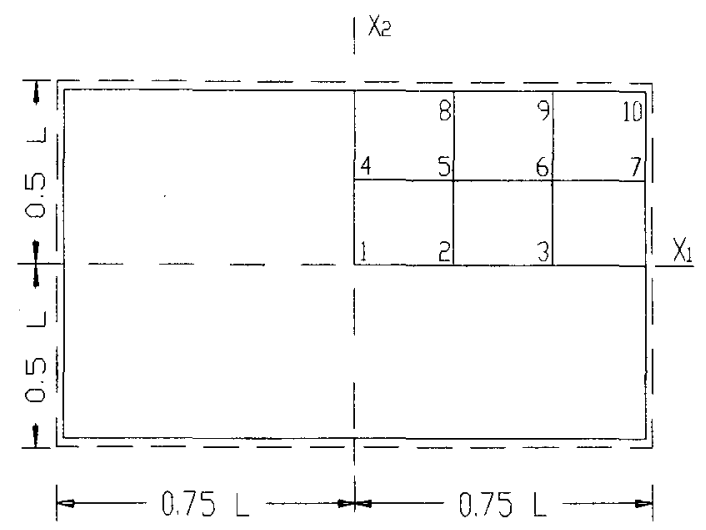

Рис.1. Прямоугольная изгибаемая пластина

Задача решается согласно математическим моделям (64) - (65), (66) - (67), Ігриняв, что дисторсия $\mathbf{q}=\Theta_{p}$. Упругие моменты $\mathbf{M}_{e f}$ представлены вектором:

$$
\begin{aligned}
& \mathbf{M}_{e f} \equiv\left\{M_{11,1}, M_{22,1}, M_{11,2}, M_{22,2}, M_{11,3}, M_{22,3}, M_{11,4}, M_{22,4}, M_{11,5}, M_{22,5} M_{12,5}, M_{11,6},\right. \\
& \left.M_{22,6}, M_{12,6}, M_{12,7}, M_{12,8}, M_{12,9}, M_{12,10}\right\}^{T} \equiv \\
& \equiv\{0.8973,1.4525,0.8628,1.3030,0.6728,0.8311,0.6642,1.1227,0.6409,1.0142 \\
& \quad-0.1931,0.5096,0.6624,-0.3800,-0.4780,-0.2770,-0.5571,- \\
& 0.7548\}^{T} .
\end{aligned}
$$

Решением нелйнейной задачи анализа (64) - (65), когда привлекаются условия текучести Мизеса, определены моменты

$$
\begin{aligned}
& \mathbf{M}_{r q}^{*} \equiv\left\{\begin{array}{lllllllllll}
-0.0860 & -0.3345 & -0.0561 & -0.1838 & -0.1622 & 0.3210 & 0.1929 & -0.2807 & 0.2296 & - \\
0.1300 & -0.0837 & 0.2001 & 0.1946 & 0.0285 & -0.0993 & -0.3003 & -0.02018 & 0.1775
\end{array}\right\}^{T} .
\end{aligned}
$$

Результатами решения задачи (66) - (67) являются векторы пластических множителей $\lambda^{*}$, моментов $\mathbf{M}_{r q}^{*}$ и искомых перемещений: 
$\mathbf{u}_{r q}^{*} \equiv\left\{\begin{array}{llllll}14.994 & 12.206 & 5.8735 & 7.3118 & 7.1601 & 4.4829\end{array}\right\}^{T}$.

Применяя формулу $\mathbf{M}_{r}^{*}=\mathbf{M}_{r q}^{*}-\mathbf{M}_{e q}$, можно определить ьстаточные моменты пластины. Для этого моменты $\mathbf{M}_{e q} \equiv\{-0.0850,-0.2900,-0.0597,-0.1423,-$ $0.1190,0.2034,0.0670,-0.1801,0.08057,-0.07508,-0.1352,0.1034,0.1024$, $-0.0500,-0.1113,-0.0971,-0.0343,0.1583\}^{\mathrm{T}}$ рассчитываются согласно формуле $\mathbf{S}_{e q}=[\bar{G}] \mathbf{q}$. Аналогично рассчитываются остаточные перемещения $\mathbf{u}_{r}^{*}$.

\section{Литература}

1. А.А. Чирас. Строительная механика. Москва: Стройиздат, 1989. 255 с.

2. W.Nowacki. Teoria sprężystości. Warszawa: PWN, 1970.

3. Ю.Ю.Аткочюнас. Математическая модель задачи анализа упруго-пластической системы в состоянии приспособляемости//Прикладная механика, 1985, 21, 79-85 c.

4. A.Borkowski. Statyzna analyza ukladów prętowych w zakresach sprężystym i plastycznym Warszawa-Poznań: PWN, 985). $200 \mathrm{~s}$.

5. Ю.Ю.Аткочюнас. Расчет упругопластических систем при повторных нагружениях. Вильнюс: Издательство науки и энциклопедий, 1994. 148 с.

6. W.T.Koiter. General Theorems for Elastic-Plastic Solids. Amsterdam: North-Holland publishing company, $1960.78 \mathrm{p}$.

7. A.Cyras. Mathematical Models for the Analysis and Optimization of Elastoplastic Structures. New York : Wiley, 1983.

8. P.G.Hodge, Jr. and T.Belytschko. Numerical Methods for the Limit Analysis of Plates // J. Appl. Mech., 1968, vol. 35, p.796-802.

9. E.Facioli and E.Vitiello. A finite element, linear programming method for the limit analysis of thin plates //Int. J. Num. Meth. Eng., 1973, vol.5, p. 311-325.

10. R.H.Gallagher. Finite Element Analysis. Prentice-Hall, Inc., Englewood Cliffs, New Jersey, 1975. $428 \mathrm{p}$.

11. Nguyen Dang, H., Köning J.A. A finite element formulation for shakedown problems using a yield criterion of the mean // Comput. Methods Appl. Mech. Eng., 1976, vol. 8, p.179-192.

12. С.Каланта. Двойственные математические модели задач огтимизации жесткопластических систем конечных элементов//Строит. механика и расчет сооружений, 1983, No3, 11-15 c. 
13. S.Kalanta. Tiesioginiai pusiausvirujų baigtinių elementų metodo algoritmai // Lietuvos skaičiuojamosios mechanikos III-iojo seminaro darbai. Vilnius: Technika, 1994, 75-81p. (Ha лит. языке).

14. Ю.Ю.Аткочюнас, А.А.Крутинис. Дисторсия в строительной механике стержневых систем //Известия вузов. Строительство и архитектура, 1988, No12, 34-37c.

15. Ю.Ю.Аткочюнас. Дисторсия в равновеснбх конечнбх злементах упругих систем //Механика деформируемых систем (Науч. тр. вузов Лит. ССР. Литовский механический сборник) 1988, No31, 7-19c.

16. J.Atkociūnas, ANorkus, L.Rimkus. Distorsija tampriuju-plastiniu strypiniu konstrukcijų mechanikoje // Naujos statybinès medžiagos, konstrukcijos ir technologijos. Vilnius: Technika, 1995, 243-248p. (на лит. языке).

17. Р.П.Каркаускас, В.Ю.Скаржаускас, Ю.Ю.Аткочпонас. Анализ неголономнопо поведения конструхщий. Вињнюс: Техника, 1995, 266-270с.

18. Ю.Ю.Аткочюнас, С.А.Каланта. Двойственный конечный элемент в задачах приспособляемости конструктий//Сопротивление материалов и теория сооружений: Респ. межвед. научпо-техн. сборник. 1986, вњII. 49. Киев: 61-63с.

19. J.Atkociūnas and ANorkus. Method of Fictitious System for Evaluation of Frame Shakedown displacements // Computers \& Structures, 1994, vol. 50, Nr.4, p. 563-567.

\section{DISTORSION IN STRUCTURAL MECHANICS}

\section{J.Atkočiūnas}

Su m mary

The behaviour of elastic perfectly plastic structures under given loadings and distorsion is examined until plastic failure (analysis problem). "Distorsion" is here for the strain which does not satisfy the compatibility equations. A distorsion can be caused by prestressing, supports displacements or lack of precision during fabrication stage (the plastic strain is the example of distorsion, too). The mathematical models for analysis problems are created by the help of extremum energy principles - principles of complementary energy and total potential energy minimum The emphasis is put on traditional to the structural mechanics bar and plate bending systems. The main unknowns are the self-equilibrium forces, displacements and strains. In the case of bar systems the analysis problem is formulated by means of linear yielding conditions as a quadratic programming problem. The analysis of Lagrange's generalised problem shows that the displacements and forces from given distorsions and plastic strains should be obtained by the same expressions. For the algoritmisation of Lagrange's problem different methods can be used: finite difference or finite element methods. Very convenient are well-known procedures of the equilibrium finite elements (with forces as main unknowns; the conditions of equilibrium satisfied) for building of stiffness and flexibility matrices. In the present paper only on the estimation of distorsion in the self-equilibrium finite elements and in the structural shakedown problems are given. The numerical example of plate analysis is presented. 